\title{
Age-dependent effect of Alzheimer's risk variant of CLU on EEG alpha rhythm in non-demented adults
}

\author{
Natalya Ponomareva ${ }^{\text {* }}$, Tatiana Andreeva ${ }^{2,3}$, Maria Protasova ${ }^{2}$, Lev Shagam ${ }^{2}$, Daria Malina ${ }^{1}$, \\ Andrei Goltsov ${ }^{2}$, Vitaly Fokin ${ }^{1}$, Andrei Mitrofanov ${ }^{4}$ and Evgeny Rogaev ${ }^{2,3,5}$ * \\ ${ }^{1}$ Brain Research Department, Research Center of Neurology Russian Academy of Medical Science, Moscow, Russia \\ ${ }^{2}$ Vavilov Institute of General Genetics, Russian Academy of Sciences, Moscow, Russia \\ ${ }^{3}$ Center of Brain Neurobiology and Neurogenetics, Institute of Cytogenetics and Genetics, Russian Academy of Sciences, Novosibirsk, Russia \\ ${ }^{4}$ Institute of Psychiatry, Moscow, Russia \\ ${ }^{5}$ University of Massachusetts Medical School, Department of Psychiatry, BNRI, Worcester, MA, USA
}

\section{Edited by:}

Davide V. Moretti, Istituto Di Ricovero e Cura a Carattere Scientifico, Italy

\section{Reviewed by:}

Bogdan O. Popescu, University Hospital Bucharest, Romania Maria Eugenia Lopez, Centre for Biomedical Technology, Spain

*Correspondence:

Natalya Ponomareva, Brain Research Department, Research Center of Neurology Russian Academy of Medical Science, Obucha-by-street 5 , 105064 Moscow, Russia e-mail: ponomare@yandex.ru; Evgeny Rogaev, Vavilov Institute of General Genetics, Russian Academy of Sciences, Moscow, Russia. Center of Brain Neurobiology and Neurogenetics, Institute of Cytogenetics and Genetics, Russian Academy of Sciences, Novosibirsk,

Russia. University of Massachusetts Medical School, Department of Psychiatry, BNRI, 303 Belmont Street, Worcester, MA 01604, USA e-mail: Evgeny.Rogaev@umassmed.edu
Polymorphism in the genomic region harboring the CLU gene (rs11136000) has been associated with the risk for Alzheimer's disease (AD). CLU C allele is assumed to confer risk for $A D$ and the allele $T$ may have a protective effect. We investigated the influence of the AD-associated CLU genotype on a common neurophysiological trait of brain activity (resting-state alpha-rhythm activity) in non-demented adults and elucidated whether this influence is modified over the course of aging. We examined quantitative electroencephalography (EEG) in a cohort of non-demented individuals (age range 20-80) divided into young (age range 20-50) and old (age range 51-80) cohorts and stratified by $C L U$ polymorphism. To rule out the effect of the apolipoprotein $E$ (ApoE) genotype on EEG characteristics, only subjects without the ApoE $\varepsilon 4$ allele were included in the study. The homozygous presence of the AD risk variant CLU CC in non-demented subjects was associated with an increase of alpha3 absolute power. Moreover, the influence of $C L U$ genotype on alpha3 was found to be higher in the subjects older than 50 years of age. The study also showed age-dependent alterations of alpha topographic distribution that occur independently of the $C L U$ genotype. The increase of upper alpha power has been associated with hippocampal atrophy in patients with mild cognitive impairment (Moretti et al., 2012a). In our study, the CLU CC-dependent increase in upper alpha rhythm, particularly enhanced in elderly non-demented individuals, may imply that the genotype is related to preclinical dysregulation of hippocampal neurophysiology in aging and that this factor may contribute to the pathogenesis of $A D$.

Keywords: Alzheimer's disease, aging, clusterin, genetic predisposition, EEG, alpha rhythm

\section{INTRODUCTION}

Alzheimer's disease $(\mathrm{AD})$ is the major cause of dementia in the elderly. It is estimated that 35.6 million people worldwide currently suffer from dementia, with the prevalence projected to increase to 65.7 million by 2030 and 115.4 million by 2050. Two-thirds of these people will likely develop $\mathrm{AD}$ (http://www.alz.co.uk/research/files/WorldAlzheimerReportExecutiveSummary.pdf). The incidence and prevalence of $\mathrm{AD}$ begins to rise as individuals reach the age of 65 , so that by the time they are in their 80 s and 90s the risk of clinical dementia is nearly $50 \%$.

Alzheimer's disease has a strong genetic basis with heritability estimates of up to $80 \%$ (Gatz et al., 2006). Mutations in the amyloid precursor protein gene (chr21), presenilin 1 (chr14), and presenilin 2 (chrl) genes are causative factors for familial AD (Goate et al., 1991; Levy-Lahad et al., 1995; Rogaev et al., 1995; Sherrington etal., 1995). A common polymorphism in the apolipoprotein E gene (ApoE), located on chromosome 19, has been established as the most common genetic risk factor for $\mathrm{AD}$ in Caucasian ethnic groups, including the Russian population
(Saunders et al., 1993; Schmechel et al., 1993; Farrer et al., 1997; Rogaev, 1999).

Recent genome-wide association studies (GWAS) studies have provided evidence that polymorphisms of the clusterin $(C L U)$ (chr8) and PICALM (chr11) genes are also associated with AD risk (Harold etal., 2009; Lambert et al., 2009; Golenkina et al., 2010). Carriers of the $C L U$ rs $1113600 C$ allele have 1.16 greater odds of developing late-onset $\mathrm{AD}$ than carriers of the potentially protective $T$ allele. Although the $\mathrm{AD}$-association with $C L U$ polymorphism alone was not confirmed in some studied populations, the putative epistatic interaction of the $C L U$ genotype with $A P O E$ $\varepsilon 4$ in risk for $\mathrm{AD}$ has been demonstrated (Golenkina et al., 2010). Approximately $36 \%$ of Caucasians carry two copies of the riskconferring allele (Bertram et al., 2007), which imply significance of this gene for public health.

The $C L U$ gene encodes glycoprotein clusterin, also known as apolipoprotein J, which shares several properties with ApoE. Clusterin and ApoE both act as amyloid- $\beta(A \beta)$ chaperones to alter $A \beta$ aggregation and/or clearance (Killick et al., 2012; Ling et al., 2012). Clusterin and ApoE are involved in the transport of cholesterol 
and phospholipids, and modulate AD-related pathways such as inflammation and apoptosis (Bettens et al., 2012; Ling et al., 2012). Clusterin is upregulated during different physiological and pathological states, such as senescence, type- 2 diabetes mellitus, AD, and in various neoplasms (Song et al., 2012; Tang et al., 2013).

In order to identify early preclinical markers for $\mathrm{AD}$, it is vital to find specific genotype-phenotype characteristics in individuals with hereditary risk for $\mathrm{AD}$ at different stages of the pathological process, including the preclinical period. Such biomarkers can be helpful for estimating the effect of potential therapies for preventing or delaying onset of neurodegenerative diseases (Illarioshkin et al., 2004; Feigin et al., 2007; Masdeu et al., 2012; Suslina, 2012). At present, there is still a mismatch between the known genetic factors of $\mathrm{AD}$, and the biomarkers reflecting the development of the pathological process.

Electroencephalography (EEG) patterns are considered to be valuable as an endophenotype - a more basic biological trait that more directly reflects the influence of the genome (Gottesman and Gould, 2003). The heritability of EEG patterns has been shown to be in the range 70-90\% (van Beijsterveldt et al., 1996). Multiple genes may modulate the alpha phenotype. Recent studies indicated that the catechol-O-methyl transferase (COMP) genotype and the gene encoding gamma-aminobutyric acid $\mathrm{B}\left(\mathrm{GABA}_{\mathrm{B}}\right)$ receptor both influence alpha voltage (Enoch et al., 2003; Winterer et al., 2003; Bodenmann et al., 2009).

Testing the association of the AD risk alleles with EEG endophenotypes can help understand where in the brain, in which stage, and during what type of information processing the genetic variant has a role.

Quantitative EEG ( $\mathrm{qEEG}$ ) has been shown to be a reliable diagnostic tool in dementia research (Stam et al., 2003; Jeong, 2004; Babiloni etal., 2006b, 2011a, 2014; Dauwels et al., 2010; Moretti et al., 2012a). Slowing of EEG in AD is a uniform finding. Patients with mild AD are characterized by higher delta and theta, and lower alpha and beta power than normal elderly subjects (Huang et al., 2000; Lizio et al., 2011). In patients with mild cognitive impairment (MCI), which is considered to be a prodromic stage of AD, EEG parameters have presented magnitudes intermediate between those observed in normal subjects and in AD patients (Babiloni et al., 2006b). Longitudinal studies have revealed qEEG-based predictors of future decline in patients with MCI and even in normal elderly subjects (Prichep et al., 2006; Van der Hiele et al., 2008; Babiloni et al., 2011b).

Alterations of alpha rhythm in particular were found to be related to $\mathrm{AD}$ development. In a resting-state condition, posterior alpha rhythms showed a power decrement in patients with MCI as compared with healthy elderly subjects (Huang et al., 2000; Jelic et al., 2000; Koenig et al., 2005; Babiloni et al., 2006b, 2014). It has been reported that, in contrast to the decrease of alpha1 $(6.9-8.9 \mathrm{~Hz})$ and alpha2 $(8.9-10.9 \mathrm{~Hz})$ relative power, the alpha3 $(10.9-12.9 \mathrm{~Hz})$ relative power increased in patients with MCI (Moretti et al., 2007, 2011, 2012a,b).

Recent studies have demonstrated the association between the $\mathrm{AD}$ genetic risk variant $A p o E \varepsilon 4$ and $\mathrm{EEG}$ in patients with $\mathrm{AD}$, MCI, and healthy subjects (Jelic et al., 1997; Lehtovirta et al., 2000; Babiloni et al., 2006b; Ponomareva et al., 2008, 2012; Lee etal., 2012). It was shown that $\mathrm{AD}$ patients carrying the $A p o E$ $\varepsilon 4$ genotype have lower alpha power and lower alpha coherence as compared to non-carriers (Jelic et al., 1997; Lehtovirta et al., 2000; Ponomareva et al., 2008). Similarly, alpha1 and alpha2 sources in occipital, temporal and limbic areas as examined by LORETA was demonstrated to have lower amplitude in $\mathrm{AD}$ and MCI patients with $A p o E \varepsilon 4$ genotype compared with those noncarrying ApoE $\varepsilon 4$ (Babiloni et al., 2006b). The authors suggested that these neurophysiological abnormalities might reflect greater impairment of the cholinergic basal forebrain, hippocampal, and thalamocortical networks. In young healthy women, Lee et al. (2012) noticed a consistent trend across the brain, in which $A p o E \& 4$ carriers possessed lower regional power at the alpha band.

The effect of CLU polymorphism on EEG characteristics has not been previously investigated, although several morphofunctional alterations associated with the $C L U$ gene risk variant were recently identified. Young healthy carriers of CLU C allele demonstrated lower white matter integrity in multiple brain regions, including several which are known to degenerate in AD (Braskie et al., 2011). Elderly cognitively normal carriers of the CLU risk allele showed significant dose-dependent longitudinal increases in resting-state regional cerebral blood flow ( $\mathrm{rCBF}$ ) in the brain regions intrinsic to memory processes, and faster rates of decline in verbal memory performance scores (Thambisetty et al., 2013). EEG activity and alpha rhythm in particular are closely related to the rCBF (Jann et al., 2010).

The purpose of this study was to examine the possible effects of the $C L U$ genotype on resting-state alpha activity in non-demented adults and to estimate whether this effect is modified over the course of aging.

We tested the hypothesis that healthy adult carriers of the AD risk variant $C L U C$ (homozygous CLU CC genotype) would show age-dependent alpha-rhythm alterations relative to carriers of the protective $T$ allele (heterozygous $C L U C T$ and homozygous $C L U$ $T T$ genotypes).

\section{MATERIALS AND METHODS PARTICIPANTS}

The enrolled cohort included 87 non-demented individuals (33 men and 54 women, age range $20-80$ years). All subjects were of Russian origin from Moscow and the Moscow region. Participants underwent a neurological examination and cognitive screening. The recruited subjects were free of dementia and other medical, psychiatric, and neurological conditions. Exclusion criteria included a personal history of mental illness, signs of clinical depression or anxiety, physical brain injury, neurological disorder, or other medical condition (e.g., hypertension, diabetes, cardiac disease, and thyroid disease), and a personal history of drug or alcohol addiction. The Spielberger state-trait anxiety inventory (Spielberger, 1983) and Hamilton rating scale for depression (Hamilton, 1960) were used to examine anxiety and depression. Subjects were evaluated with the mini-mental state examination (MMSE) and Clinical Dementia Rating (CDR) scale (Hughes et al., 1982). Only subjects with MMSE scores of 28 and more and CDR scale 0 cases were included in the study. All subjects were righthanded. 
Informed written consent was obtained from all participants. The experimental protocol of this study was approved by the local Ethics Committee.

ApoE genotyping was performed on all participants, and to exclude the effect of the ApoE genotype on EEG characteristics, only subjects without the $A p o E \varepsilon 4$ allele were included in the study.

All subjects were divided into subgroups according to $C L U$ (CLU CC and CLUCT\&TT) polymorphism. The homozygous $C L U C C$ group included subjects with two $C$ alleles of $C L U$, and the $C L U C T \& T T$ group consisted of subjects with heterozygous CLU CT or homozygous CLU TT genotypes. The participants with $C L U C C$ as well as with CLU CT\&TT genotypes were also divided into cohorts of those younger and older than 50 years of age.

\section{EEG RECORDING}

All recordings were obtained in the afternoon at 3-4 pm. During the experiments, the subjects sat comfortably in a chair. They were asked to close their eyes and to relax during the recording. The technician watched the subject's vigilance state continuously by monitoring the EEG and observing the subject.

The registration and evaluation of EEG has been carried out in accordance with the International Pharmaco-EEG Society (IPEG) guidelines (Versavel et al., 1995; Jobert et al., 2012). EEGs were recorded during resting with eyes closed on a Nihon Kohden 4217 G EEG using a time constant of $0.3 \mathrm{~s}$. The $16 \mathrm{Ag} / \mathrm{AgCl}$ electrodes were placed according to the international 10-20 system at $\mathrm{O} 2, \mathrm{O} 1$, P4, P3, C4, C3, F4, F3, Fp2, Fp1, T6, T5, T4, T3, F8, and F7 positions. Linked ears served as the reference. Electrode impedance did not exceed $10 \mathrm{k} \Omega$. During the recording, $180 \mathrm{~s}$ of EEG in resting conditions were simultaneously sampled at $256 \mathrm{~Hz}$ and stored on a computer for further analysis off-line. The records were digitally filtered with a band-pass filter of $1.0-45.0 \mathrm{~Hz}$ prior to analysis. Periods of artifact were eliminated from subsequent analysis. Identification and removal of artifacts (ocular, cardiac, muscular, sweating and respiratory, electrode movements) were performed by two expert electroencephalographists (P.N.V., M.D.D.) in accordance with criteria thoroughly described elsewhere (Moretti et al., 2003; Tatum et al., 2011; Jobert et al., 2012).

\section{DATA ANALYSIS}

Thirty-six to forty artifact-free 4-s epochs of resting EEG were processed by fast Fourier transform. Absolute power for the frequencies of interest: alpha1 (7.5-8.99), alpha2 (9.00-10.99), alpha3 (11-12.99), and for the regions of interest (ROI): occipital (O2, O1), frontal 1 (F4, F3), frontal 2 (Fp2, Fp1), temporal 1 (T6, T5), and temporal 2 (T4, T3) were calculated.

These alpha band frequencies were chosen by averaging those used in previous relevant EEG studies on aging, genetic influences, and dementia (Babiloni et al., 2006a,b; Bodenmann et al., 2009; Moretti et al., 2012a,b). This allowed better comparison of our results with the previous literature on aging and genetics, but it did not account for individual alpha frequencies peak (Klimesch, 1999).

Log transformations of the absolute power of the various bandwidths in each derivation were calculated in order to compensate for data skewness, as recommended by John et al. (1980).

\section{GENETIC ANALYSIS}

Genomic DNA was isolated from peripheral venous blood by the standard phenol-chloroform extraction methodology, or by using a Qiagen kit for DNA isolation. Genotyping was performed by polymerase chain reaction (PCR) and followed by restriction fragment length polymorphism (RFLP) analysis. Amplification was performed according to the manufacturer's instructions using both the Tercyc DNA amplifier (DNA technology, Russia) and the GeneAmp PCR System 9700 Thermal Cycler (Applied Biosystems).

To genotype the APOE gene locus, the following oligonucleotide primers were used: $5^{\prime}$ _CGGCTGGGCGCG_GACATGGAGGA and $5^{\prime}$ _TCGCGGGCCCCGGC_CTGGTACAC. The PCR protocol was as follows: preliminary denaturation at $95^{\circ} \mathrm{C}$ for $4 \mathrm{~min}$; 5 cycles: $95^{\circ} \mathrm{C}$ for $45 \mathrm{~s}, 54^{\circ} \mathrm{C}$ for $25 \mathrm{~s}$, and $72^{\circ} \mathrm{C}$ for $30 \mathrm{~s}$; and 30 cycles: $95^{\circ} \mathrm{C}$ for $5 \mathrm{~s}, 58^{\circ} \mathrm{C}$ for $15 \mathrm{~s}$, and $72^{\circ} \mathrm{C}$ for $5 \mathrm{~s}$; the last stage was performed at $72^{\circ} \mathrm{C}$ for $3 \mathrm{~min}$. PCR products were then cleaved by HhaI or BstHHI (SibEnzyme, Russia) and restriction products were analyzed in $7.5 \%$ polyacrylamide gel.

The rs 11136000 polymorphism in CLU gene was tested with the following oligonucleotide primers: 5'_CTTTGTAATGATGTACC ATCTACCC and 5'_AGGCTGCAGACTCCCTGAAT. The PCR protocol was as follows: preliminary denaturation at $95^{\circ} \mathrm{C}$ for $1 \mathrm{~min}$ and 35 cycles: $94^{\circ} \mathrm{C}$ for $30 \mathrm{~s}, 57^{\circ} \mathrm{C}$ for $30 \mathrm{~s}$, and $72^{\circ} \mathrm{C}$ for $1 \mathrm{~min}$. The last stage was performed at $72^{\circ} \mathrm{C}$. The $645 \mathrm{bp}$ PCR products were then cleaved by AcsI restriction endonuclease (SibEnzyme, Russia) and restriction fragments were analyzed in $2 \%$ agarose gel.

\section{STATISTICS}

Differences in demographic scores between the groups (CLU CC young, CLU CT\&TT young, CLU CC old, CLU CT\&TT old) were tested using analysis of variance (ANOVA) for continuous variables (age, education), and the Mann-Whitney $U$ test for categorical variables (sex).

Electroencephalography parameters from each group were tested for the normal distribution by the Wilk-Shapiro test, and in no cases were the data skewed. The significance of the differences between the log-transformed EEG parameters was estimated using repeated measures of ANOVA in the general linear model (GLM) separately for alpha1, alpha2, and alpha3 bands, with Genotype (CLU CC vs CLU CT\&TT) and Age cohort (old vs. young) as between-subjects factors, and ROI: occipital (O2, O1), frontal 1 (F4, F3), frontal 2 (Fp2, Fp1), temporal 1 (T6, T5), temporal 2 (T4, T3), and hemisphere (right, left) as a withinsubject factor. Post hoc comparisons for between-subject effects and within-subject effects were analyzed using the Duncan test, and the level of significance was set to $P<0.05$ for post hoc comparisons.

\section{RESULTS}

Table 1 shows the demographic information for the participants. There were no differences in age and sex between the CLU CC and $C L U C T \& T T$ subgroups in either the young or the old subgroups and in the whole sample $(P>0.05)$. There were no significant differences in sex between the young and the old subgroups with the same $C L U$ genotype. 
Table 1 | Demographic characteristics of participants.

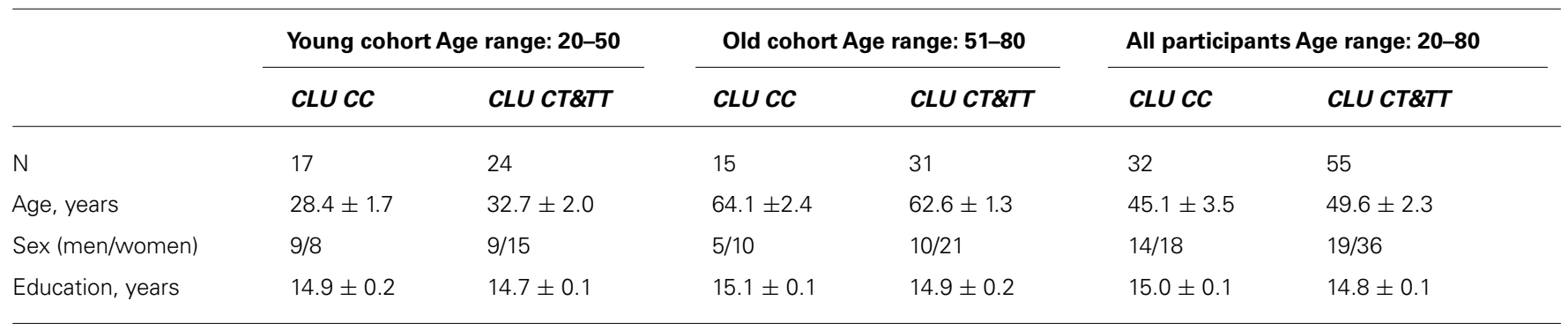

Data are presented as means and standard errors.

\section{INFLUENCE OF AGING ON TOPOGRAPHIC DISTRIBUTION AND FREQUENCY OF ALPHA ACTIVITY IN HEALTHY ADULTS}

The ANOVA revealed a significant effect of ROI on alpha1, alpha2, and alpha3 absolute power (for alpha1, $F[4,332]=111.97$, $P=0.0000$; for alpha2, $F([4,332]=195.96, P=0.0000$; for alpha3, $F[4,332]=178.36, P=0.0000)$. Post hoc comparisons showed that in the entire sample, which included young and old cohorts, absolute power was higher in occipital than in frontal and temporal regions in the alpha1, alpha2, and alpha3 bands $(P<0.0001)$. Moreover, the power of all alpha bands was higher in frontal as compared to temporal areas $(P<0.0001)$.

There was no significant statistical Age $\times$ ROI interaction effect on alpha1 power (Figure 1A), but such an effect was observed on alpha 2 and alpha 3 bands $(F[4,332]=6.33, P=0.00006$ for alpha2; $F[4,332]=15.30, P=0.00000$ for alpha3). In the old cohort, the differences in alpha2 power between the ROI were reduced. Whereas in the young cohort, alpha2 power was higher in frontal Fp than in temporal posterior Tp areas (post hoc comparisons $P=0.002)$, in the old cohort the differences in these areas were not significant $(P=0.11)$. The differences between frontal Fp and temporal posterior $\mathrm{Tp}$ areas were significantly smaller in the old than in the young cohort $(P=0.02$; Figure 1B).

Similarly, in the old cohort, the differences in alpha3 power between ROI were reduced as compared to the young cohort. In the young cohort, alpha3 power was higher in temporal posterior than in temporal areas $(P=0.02)$; in the old cohort these differences were not significant $(P=0.1)$, and age-related changes of these regional differences were also not significant. Alpha3 power was lower in the occipital ROI in the old cohort as compared to the young $(P<0.01$; Figure 1C).

A significant interaction effect between the factors Age and Bands was observed $(F[2,166]=4.51, P=0.01)$. In the young subjects, alpha 2 power was significantly higher than alpha1 and alpha3 power $(P=0.00001)$, while in the old subjects the alpha1 power tended to increase and the differences between alphal and alpha2 power were not significant (Figure 2).

\section{STATISTICAL ANALYSIS OF CLU EFFECT ON ALPHA ACTIVITY}

The results of ANOVA showed that the main effect of CLU Genotype was significant on alpha3 $(F[1,83]=5.57, P=0.021)$, but not on alpha1 $(F[1,83]=2.10, P=0.15)$ or alpha2 $(F[1,83]=2.81$, $P=0.10)$ absolute power. Post hoc comparison revealed that in the entire sample, which included old and young cohorts, alpha3 absolute power in the subjects with homozygous CLUCC genotype was significantly higher than in the subjects with heterozygous CLUCT and homozygous CLU TT (CLU CT\&TT) genotypes $(P=0.017)$. Moreover, post hoc comparison showed that, in the old cohorts, alpha3 power was significantly higher in the CLU CC than in the CLU CT\&TT carriers $(P=0.016)$, while in the young cohorts the differences in alpha3 power between the CLU CC and CLU CT\&TT carriers did not reach a significant level (Figure 3C). There were no significant differences in alpha1 and alpha2 power between the young CLU CC and CLU CT\&TT carriers (Figures 3A,B). In the old cohorts, alphal power was higher in the CLU CC than in the CLU CT\&TT carriers $(P=0.04)$, while the differences of alpha2 power in the old CLU CC and CLU CT\&TT carriers were not significant $(P=0.1$; Figures $3 \mathbf{A}, \mathbf{B})$.

Topographic analysis demonstrated that the most pronounced differences between the homozygous CLU CC and CLU CT\&TT carriers were observed in alpha3 power in the old cohorts. In the young cohorts, there were no significant differences in any ROI in alpha1, alpha2, and alpha3 power between $C L U C C$ and $C L U$ $C T \& T T$ carriers (Figures $4 \mathbf{A}-\mathbf{C}$ ). In the old cohort the differences between $C L U C C$ and CLU CT\&TT carriers were significant for alphal power in occipital $(P=0.02)$ and temporal posterior areas $(P=0.02)$, for alpha3 power - in frontal $(P=0.04)$, frontal poles $(P=0.03)$, and temporal posterior $(P=0.02)$ areas (Figures 4A,C).

A significant $C L U \times$ ROI interaction effect on the alphal power in the entire sample was observed $(F[4,332]=3.43, P=0.009)$. In the $C L U C C$ carriers alphal power was higher in occipital than in frontal areas $(P<0.0001)$ and in temporal posterior than in temporal areas $(P=0.001)$, while in the subjects with CLUCT\&TT genotypes the differences in alphal power between occipital and frontal areas were smaller $(P=0.01)$, the differences between the temporal posterior and temporal areas were not significant $(P=0.3)$. There was a tendency toward higher alphal power in all ROI in the subjects with $C L U C C$ genotype as compared to the subjects with CLU CT\&TT genotype.

\section{DISCUSSION}

The main findings of this study show that the CLU genotype exerts a significant effect on alpha absolute power in the resting-state EEG of healthy adults. The homozygous presence of the AD risk variant CLU CC in non-demented subjects was associated with an increase of alpha3 and to a lesser, though significant, extent of alphal power in the subjects older than 50 years of age. CLU genotype-related differences were also found in the topographic 


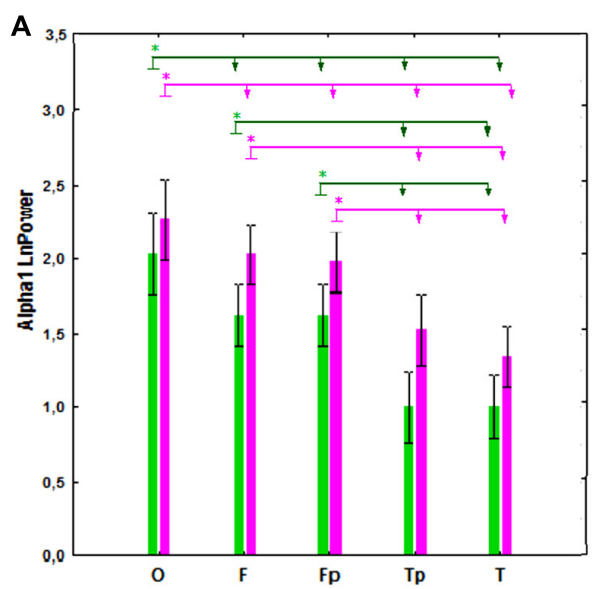

B

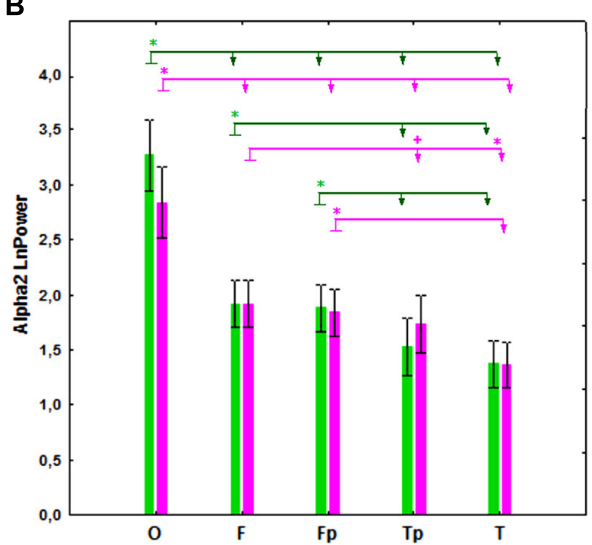

C

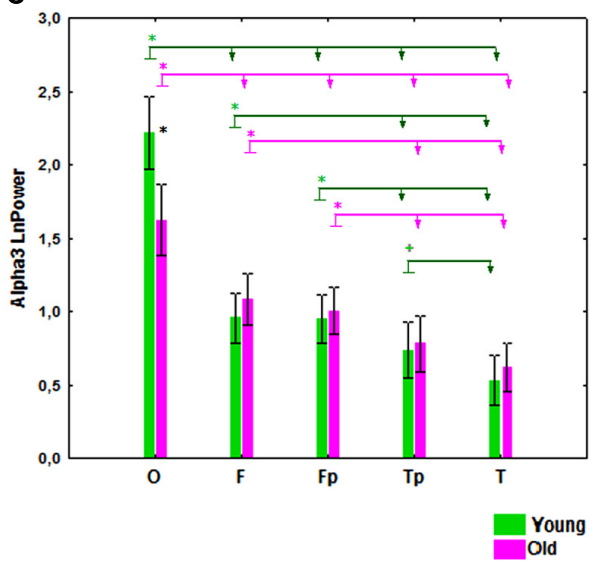

FIGURE 1 | Absolute power (mean and SE) of alpha1 (A), alpha2 (B), and alpha3 (C) bands in the young and old cohorts, for occipital (O), frontal (F), frontal poles ( $F p)$, temporal posterior $(T p)$, and temporal $(T)$ areas. Black asterisks $\left({ }^{*}\right)$ indicate a $P<0.01$ significant difference in absolute spectral power between two cohorts in the same region of interest (ROI). The arrows labeled with green (for the young cohort) and purple (for the old cohort) asterisks compare different ROI in the same cohort. The ROl at the start of the arrow has either $(+) P<0.05$ or $\left({ }^{*}\right)$ $P<0.01$ significant differences in absolute spectral power compared the ROls at the ends of the arrow.

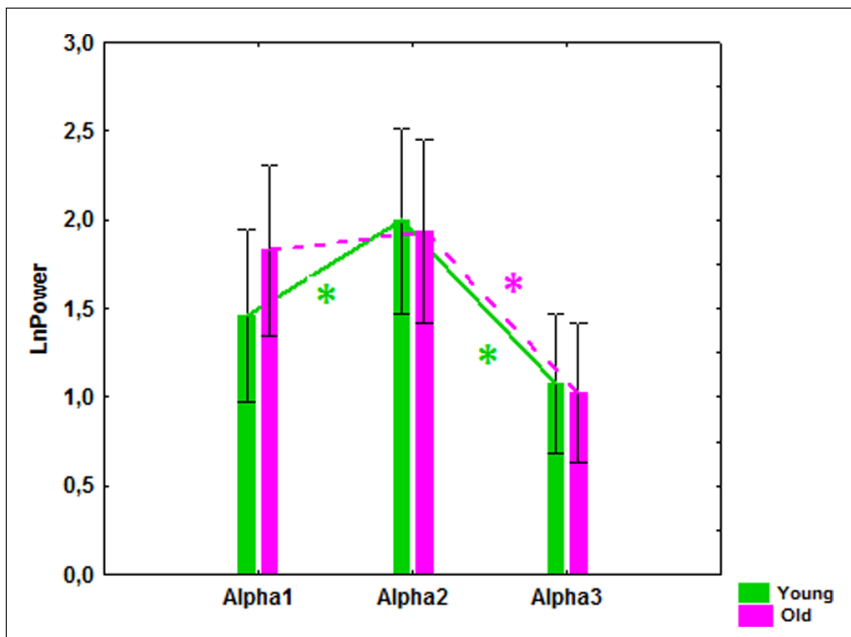

FIGURE 2 | Alpha1, alpha2, and alpha3 absolute power (mean and SE) in the healthy young and old cohorts. ${ }^{*} P \leq 0.01$, significant differences between the alpha bands in the young (green) and old (purple) cohorts.

distribution of alphal activity: in the subjects with homozygous $C L U$ CC genotype, alphal power was higher in occipital than in frontal regions, while in the subjects with heterozygous $C L U$ $C T$ and homozygous CLU TT (CLU CT\&TT) genotypes the differences in alphal power between occipital and frontal regions were not significant. The present study also showed age-related alterations of the topographic distribution of alpha2 and alpha3 activities, and an age-related increase in power of alphal relative to alpha2, all of which occurred in the subjects with CLU CC as well as with $C L U C T \& T T$ genotypes.

Alpha rhythm reflects the activity of dominant oscillatory neural networks in resting adults and represents a basic functional feature of the working brain (Klimesch, 2012). Alpha oscillations have been associated with essential cognitive functions, such as memory, intelligence quotient, internal attention (Cooper et al., 2006; Klimesch, 2012), and inhibitory control of motor programs (Pfurtscheller et al., 2000; Başar, 2012).

Inhibitory processes underlie alpha synchronization (Klimesch, 2012). During the awake resting condition, the voltage of the alpha rhythms is inversely correlated with the cortical activation. Alpha rhythm is modulated by thalamocortical and corticocortical interactions playing role in the transmission of sensorimotor information between subcortical and cortical pathways, and the retrieval of semantic information from cortical regions (Steriade and Llinás, 1988; Brunia, 1999; Pfurtscheller and Lopes da Silva, 1999).

According to prior research in this area, alpha rhythm is not a unitary phenomenon. Upper alpha $(11-13 \mathrm{~Hz})$ is more involved in cortical processes related to the semantic memory and low alpha $(8-11 \mathrm{~Hz})$ is more involved in attentional demands (Klimesch, 1999). Different neural networks have been suggested as generating low alpha and high alpha frequency bands. The modulation of the low alpha was proposed to be related to the corticosubcortical mechanisms, such as corticothalamic, corticostriatal, and corticobasal, while the upper alpha band is affected to a greater 
A

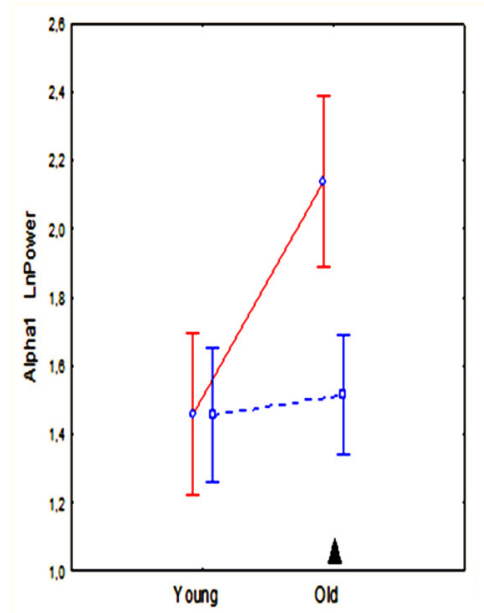

B

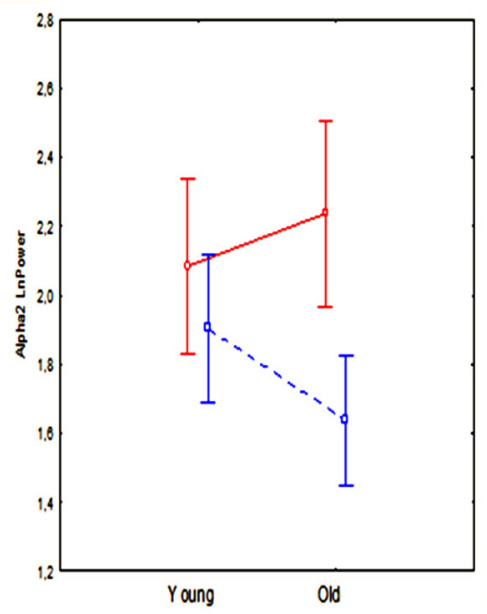

C

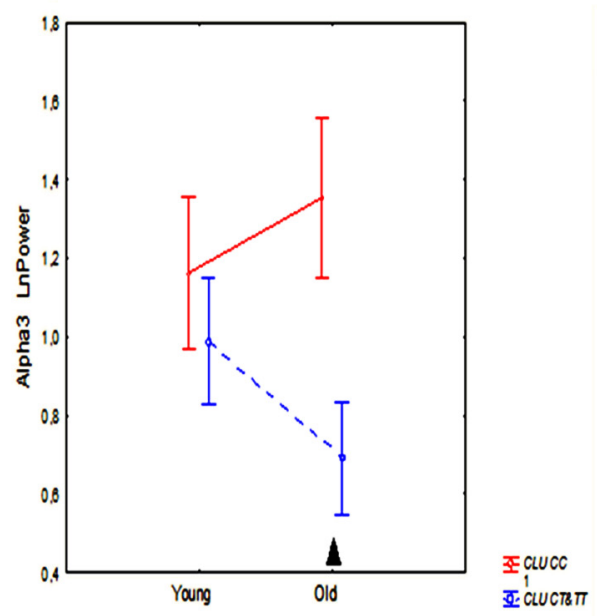

FIGURE 3 | The average absolute power of alpha1 (A), alpha2 (B), and alpha3 (C) bands (mean and SE) in young and old subjects with $C L U$ CC and CLU CT\&TT genotypes. Triangle indicates significant differences between the CLU CC and CLU CT\&TT carriers $P<0.05$.

extent by the hippocampus and other corticocortical interactions (Moretti et al., 2012a,b).

Recent study has shown an increase of the upper alpha power in patients with MCI and $\mathrm{AD}$, when compared to normal elderly subjects (Moretti et al., 2012a,b). The increase in alpha3/alpha2 ratio in frontal and temporoparietal areas was associated with hippocampal atrophy in MCI (Moretti et al., 2007). The increase of alpha3/alpha2 ratio in subjects with MCI was suggested to reflect impairment of the anterior attentive mechanisms in subjects with MCI, in spite of the absence of overt clinical deficit (Moretti et al., 2012a,b). This increase was hypothesized to be due to a removal of excitatory, synaptic cholinergic inputs in intracortical networks, which would produce a decrease in synaptic efficacy and functional disconnection of cortical circuits (Steriade, 2006).

Healthy carriers of $\mathrm{AD}$ risk variant $C L U C C$, especially old subjects with this genotype, may have similar, though less pronounced, alterations underlying the increase of upper alpha activity to those found in MCI subjects. These alterations may include the dysregulation of excitatory synaptic inputs, especially cholinergic ones, in hippocampus and frontal intracortical networks. In the old $C L U C C$ carriers we also found an increase in alphal power, though less pronounced, than in alpha3 power. These finding suggest that in the old CLU CC carriers the dysregulation may affect other mechanisms, such as corticothalamic, corticostriatal, and corticobasal ones, involved in low alpha generation (Moretti et al., 2012a,b).

Even normal aging is accompanied by a gradual loss of cholinergic function caused by dendritic, synaptic, and axonal degeneration as well as a decrease in trophic support. As a consequence, impairments in intracellular signaling and cytoskeletal transport may mediate cholinergic cell atrophy, finally leading to the known age-related functional decline in the brain, including aging-associated cognitive impairments (Schliebs and Arendt, 2011).
In line with previous studies, our results also demonstrated that in all individuals, independently of CLU genotype, aging is accompanied by changes in spectral power and topographic distribution of alpha activity (Tsuno et al., 2002; Babiloni et al., 2006a; Chiang et al., 2011). We found the decrease of alpha3 power in occipital areas, the reduction of the differences of alpha 2 and alpha 3 activity between posterior and anterior areas (anteriorization of alpha) and the trend toward the decrease of alpha2 power and increase of alpha1 power in the old cohort as compared to the young. It has been demonstrated that posterior cortical alpha rhythms decreases in magnitude during physiological aging (Babiloni et al., 2006a). A slowing of the alpha frequency peak in normal adults during physiological aging has also been reported (Klimesch, 1999). The anteriorization of alpha activity in elderly subjects was found to be related to a decreased level of vigilance (Tsuno et al., 2002). It was suggested that the anteriorization of alpha activity is related to the alterations in activation of posterior and anterior default mode networks (DMNs) and that these changes might be susceptible to dopaminergic influences (Knyazev, 2012). Chronic excessive neuronal activity during a resting-state condition in DMN can lead to $A \beta$ deposition (Bero et al., 2011; de Haan et al., 2012). On the other hand, elevated level of $A \beta$ elicits epileptiform activity, probably by enhancing synchrony among the glutamatergic synapses (Palop and Mucke, 2009). The brain regions of the DMN were shown to be preferentially vulnerable to neurodegenerative processes (Vlassenko et al., 2010; Hsiao et al., 2013).

The effect of aging on EEG is modulated by genetic factors (Babiloni et al., 2006b; Ponomareva et al., 2012). Several lines of evidence imply that the effect of CLU genotype on brain function may be observed before the onset of cognitive impairment. The CLU risk variant rs11136000 was found to be associated with reduced integrity of broad white matter regions, as observed with diffusion tensor imaging in young healthy adults (Braskie et al., 2011). fMRI study showed aberrant activation in the frontal and 


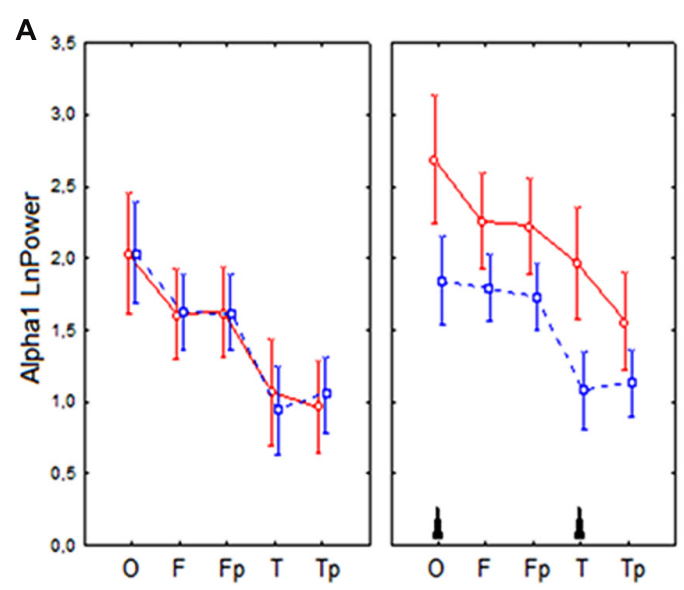

B

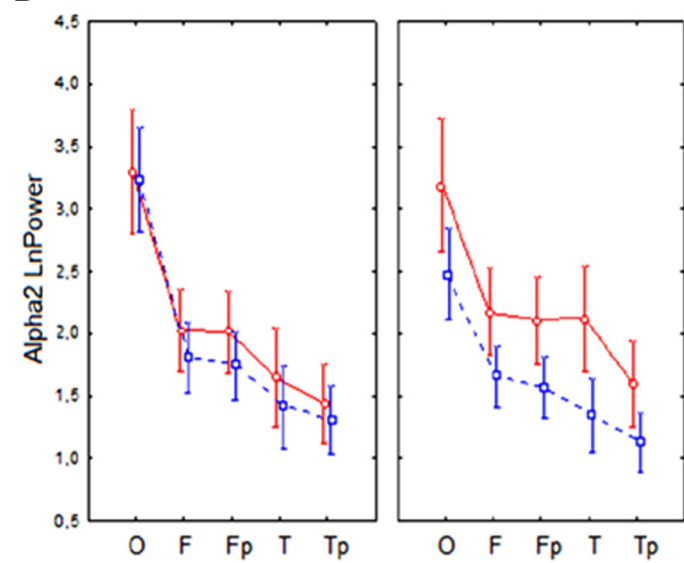

C
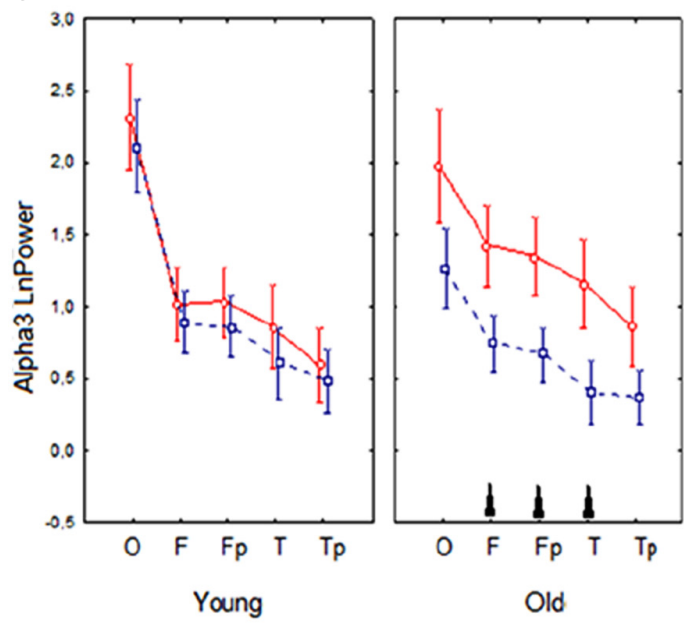

쪼 CLUCC

호. CLU CT\&TT

FIGURE 4 | Topographic distribution of alpha1 (A), alpha2 (B), and alpha3 (C) absolute power (mean and SE) in young and old carriers of $C L U C C$ and $C L U C T \& T T$ genotypes in occipital $O$, frontal $F$, frontal poles Fp, temporal posterior Tp, and temporalT areas. Triangle indicates significant differences between the CLU CC and CLU CT\&TT carriers $P<0.05$. posterior cingulate cortex and the hippocampus during working memory performance in healthy young individuals carrying $C L U$ AD risk genotype (Lancaster et al., 2011).

Recently the robust changes in $\mathrm{rCBF}$ in cognitively normal old individuals carrying the $C$-allele of the rs11136000 SNP were revealed (Thambisetty et al., 2013). These changes consisted of significant longitudinal increases in $\mathrm{rCBF}$ in the hippocampus and anterior cingulated cortex. The authors suggested that the effect of CLU CC genotype may be related to the deposition of beta amyloid, and that affected regions are vulnerable to disruption by deposition of beta amyloid, even in the non-demented elderly.

The effect of CLU genotype on resting EEG in healthy subjects was not similar to the effect of $A p o E$ genotype found in prior studies (Babiloni et al., 2006b; Lee et al., 2012). The differences are in line with the differing influence of $C L U$ and $A p o E$ genotype on resting rCBF in normal aging (Thambisetty et al., 2013). The authors reported longitudinal increase during aging of restingstate $\mathrm{rCBF}$ in the hippocampus and anterior cingulate cortex in the $C L U C C$ carriers and the decrease in resting $\mathrm{rCBF}$ in the frontal, parietal, and temporal cortices and its increases in the insular cortex in the old ApoE $\varepsilon 4$ carriers.

Alpha rhythm slowing was found to occur in aging, and the alpha1 band of the young group might have some functional differences from the alphal band in the old subjects (Chiang et al., 2011). This is a potential limitation of our study. However, as $C L U$ genotype-related differences were found in the age-adjusted groups, this possible confounding factor could not affect the results concerning the influence of CLU genotype on alpha power.

\section{CONCLUSION}

Our results show that the presence of the homozygous CLU $\mathrm{CC}, \mathrm{AD}$ risk variant, is associated with increased absolute power of alpha3 activity and changes in topographical distribution of alphal activity and that this effect is more pronounced in the subjects older than 50 years of age. The increased synchronization of upper alpha activity may be related to the alterations in cholinergic hippocampal and cortical networks. The effect of CLU genotype on alpha activity can be superimposed to the other EEG alterations that occur across physiological aging.

\section{ACKNOWLEDGMENTS}

Research was supported by Grants from the Government of the Russian Federation (No 14.B25.31.0033), RFBR No 11-04-01896a, and in part by the Ministry of Education and Science of the Russian Federation (Contract No 8053), RFBR (11-04-02106-a), Rosbiolab and NIH/NIA AG029360. We also thank Chikunov A. V. for support.

\section{REFERENCES}

Babiloni, C., Binetti, G., Cassarino, A., Dal Forno, G., Del Percio, C., Ferreri, F. et al. (2006a). Sources of cortical rhythms in adults during physiological aging: a multicentric EEG study. Hum. Brain Mapp. 27, 162-172. doi: 10.1002/hbm.20175

Babiloni, C., Benussi, L., Binetti, G., Cassetta, E., Dal Forno, G., Del Percio, C., et al. (2006b). Apolipoprotein E and alpha brain rhythms in mild cognitive impairment: a multicentric electroencephalogram study. Ann. Neurol. 59, 323-334. doi: 10.1002/ana.20724

Babiloni, C., Del Percio, C., Lizio, R., Marzano, N., Infarinato, F., Soricelli, A., etal. (2014). Cortical sources of resting state electroencephalographic alpha rhythms deteriorate across time in subjects with amnesic mild cognitive 
impairment. Neurobiol. Aging 35, 130-142. doi: 10.1016/j.neurobiolaging.2013. 06.019

Babiloni, C., Vecchio, F., Lizio, R., Ferri, R., Rodriguez, G., Marzano, N., et al. (2011a). Resting state cortical rhythms in mild cognitive impairment and Alzheimer's disease: electroencephalographic evidence. J. Alzheimers Dis. 26(Suppl. 3), 201-214. doi: 10.3233/JAD-2011-0051

Babiloni, C., Lizio, R., Carducci, F., Vecchio, F., Redolfi, A., Marino, S., etal. (2011b). Resting state cortical electroencephalographic rhythms and white matter vascular lesions in subjects with Alzheimer's disease: an Italian multicenter study. J. Alzheimers Dis. 26, 331-346. doi: 10.3233/JAD-2011101710

Başar, E. (2012). A review of alpha activity in integrative brain function: fundamental physiology, sensory coding, cognition and pathology. Int. J. Psychophysiol. 86, 1-24. doi: 10.1016/j.ijpsycho.2012.07.002

Bero, A. W., Yan, P., Roh, J. H., Cirrito, J. R., Stewart, F. R., Raichle, M. E., et al. (2011). Neuronal activity regulates the regional vulnerability to amyloid- $\beta$ deposition. Nat. Neurosci. 14, 750-756. doi: 10.1038/nn.2801

Bertram, L., McQueen, M. B., Mullin, K., Blacker, D., and Tanzi, R. E. (2007). Systematic meta-analyses of Alzheimer disease genetic association studies: the AlzGene database. Nat. Genet. 39, 17-23. doi: 10.1038/ ng1934

Bettens, K., Brouwers, N., Engelborghs, S., Lambert, J. C., Rogaeva, E., Vandenberghe, R., et al. (2012). Both common variations and rare non-synonymous substitutions and small insertion/deletions in CLU are associated with increased Alzheimer risk. Mol. Neurodegener. 7, 3. doi: 10.1186/1750-1326-7-3

Bodenmann, S., Rusterholz, T., Dürr, R., Stoll, C., Bachmann, V., Geissler, E., et al. (2009). The functional Val158Met polymorphism of COMT predicts interindividual differences in brain alpha oscillations in young men. J. Neurosci. 29, 10855-10862. doi: 10.1523/JNEUROSCI.1427-09.2009

Braskie, M. N., Jahanshad, N., Stein, J. L., Barysheva, M., McMahon, K. L., de Zubicaray, G. I., et al. (2011). Common Alzheimer's disease risk variant within the CLU gene affects white matter microstructure in young adults. J. Neurosci. 31, 6764-6770. doi: 10.1523/JNEUROSCI.5794-10.2011

Brunia, C. H. (1999). Neural aspects of anticipatory behavior. Acta Psychol. (Amst.) 101, 213-242. doi: 10.1016/S0001-6918(99)00006-2

Chiang, A. K., Rennie, C. J., Robinson, P. A., van Albada, S. J., and Kerr, C. C. (2011). Age trends and sex differences of alpha rhythms including split alpha peaks. Clin. Neurophysiol. 122, 1505-1517. doi: 10.1016/j.clinph.2011.01.040

Cooper, N. R., Burgess, A. P., Croft, R. J., and Gruzelier, J. H. (2006). Investigating evoked and induced electroencephalogram activity in task-related alpha power increases during an internally directed attention task. Neuroreport 17, 205-208. doi: 10.1097/01.wnr.0000198433.29389.54

Dauwels, J., Vialatte, F., and Cichocki, A. (2010). Diagnosis of Alzheimer's disease from EEG signals: where are we standing? Curr. Alzheimer Res. 7, 487-505. doi: $10.2174 / 156720510792231720$

de Haan, W., Mott, K., van Straaten, E. C., Scheltens, P., and Stam, C. J. (2012). Activity dependent degeneration explains hub vulnerability in Alzheimer's disease. PLoS Comput. Biol. 8:e1002582. doi: 10.1371/journal.pcbi.10 02582

Enoch, M. A., Xu, K., Ferro, E., Harris, C. R., and Goldman, D. (2003). Genetic origins of anxiety in women: a role for a functional catechol-O-methyltransferase polymorphism. Psychiatr. Genet. 13, 33-41. doi: 10.1097/00041444-20030300000006

Farrer, L. A., Cupples, A., Haines, J. L., Hyman, B., Kukull, W. A., Mayeux, R., et al. (1997). Effects of age, sex, and ethnicity on the association between apolipoprotein E genotype and Alzheimer's disease. A meta-analysis. APOE and Alzheimer Disease Meta Analysis Consortium. JAMA 278, 1349-1356. doi: 10.1001/jama.1997.03550160069041

Feigin, A., Tang, C., Ma, Y., Mattis, P., Zgaljardic, D., Guttman, M., et al. (2007). Thalamic metabolism and symptom onset in preclinical Huntington's disease. Brain 130, 2858-2867. doi: 10.1093/brain/awm217

Gatz, M., Reynolds, C. A., Fratiglioni, L., Johansson, B., Mortimer, J. A., Berg, S., et al. (2006). Role of genes and environments for explaining Alzheimer disease. Arch. Gen. Psychiatry 63, 168-174. doi: 10.1001/archpsyc.63.2.168

Goate, A., Chartier-Harlin, M. C., Mullan, M., Brown, J., Crawford, F., Fidani, L., etal. (1991). Segregation of missense mutation in the amyloid precursor protein gene with familial Alzheimer's disease. Nature 349, 704-706. doi: $10.1038 / 349704 a 0$
Golenkina, S. A., Goltsov, A. Yu., Kuznetsova, I. L., Grigorenko, A. P., Andreeva, T. V., Reshetov, D. A., et al. (2010). Analysis of clusterin gene (CLU/APOJ) polymorphism in Alzheimer's disease patients and in normal cohorts from Russian populations. Mol. Biol. 44, 620-626. doi: 10.1134/S00268933100 40072

Gottesman, I. I., and Gould, T. D. (2003). The endophenotype concept in psychiatry: etymology and strategic intentions. Am. J. Psychiatry 160, 636-645. doi: 10.1176/appi.ajp.160.4.636

Hamilton, M. (1960). A rating scale for depression. J. Neurol. Neurosurg. Psychiatry 23, 56-62. doi: 10.1136/jnnp.23.1.56

Harold, D., Abraham, R., Hollingworth, P., Sims, R., Gerrish, A., Hamshere, M. L., et al. (2009). Genome-wide association study identifies variants at CLU and PICALM associated with Alzheimer's disease. Nat. Genet. 41, 1088-1093. doi: 10.1038/ng.440

Hsiao, F. J., Wang, Y. J., Yan, S. H., Chen, W. T., and Lin, Y. Y. (2013). Altered oscillation and synchronization of default-mode network activity in mild Alzheimer's disease compared to mild cognitive impairment: an electrophysiological study. PLoS ONE 8:e68792. doi: 10.1371/journal.pone.0068792

Huang, C., Wahlund, L., Dierks, T., Julin, P., Winblad, B., and Jelic, V. (2000). Discrimination of Alzheimer's disease and mild cognitive impairment by equivalent EEG sources: a cross-sectional and longitudinal study. Clin. Neurophysiol. 111, 1961-1967. doi: 10.1016/S1388-2457(00)00454-5

Hughes, C. P., Berg, L., Danziger, W. L., Coben, L. A., and Martin, R. L. (1982). A new clinical scale for the staging of dementia. Br. J. Psychiatry 140, 566-572. doi: 10.1192/bjp.140.6.566

Illarioshkin, S. N., Ivanova-Smolenskaia, I. A., Markova, E. D., Shadrina, M. I., Kliushnikov, S. A., Zagorovskaia, T. V., et al. (2004). Molecular genetic analysis of hereditary neurodegenerative diseases. Genetika 4, 816-826.

Jann, K., Koenig, T., Dierks, T., Boesch, C., and Federspiel, A. (2010). Association of individual resting state EEG alpha frequency and cerebral blood flow. Neuroimage 51, 365-372. doi: 10.1016/j.neuroimage.2010.02.024

Jelic, V., Johansson, S. E., Almkvist, O., Shigeta, M., Julin, P., Nordberg, A., et al. (2000). Quantitative electroencephalography in mild cognitive impairment: longitudinal changes and possible prediction of Alzheimer's disease. Neurobiol. Aging 21, 533-540. doi: 10.1016/S0197-4580(00)00153-6

Jelic, V., Julin, P., Shigeta, M., Nordberg, A., Lannfelt, L., Winblad, B., et al. (1997). Apolipoprotein E epsilon 4 allele decreases functional connectivity in Alzheimer's disease as measured by EEG coherence. J. Neurol. Neurosurg. Psychiatry 63, 59-65. doi: 10.1136/jnnp.63.1.59

Jeong, J. (2004). EEG dynamics in patients with Alzheimer's disease. Clin. Neurophysiol. 115, 1490-1505. doi: 10.1016/j.clinph.2004.01.001

Jobert, M., Wilson, F. J., Ruigt, G. S., Brunovsky, M., Prichep, L. S., and Drinkenburg, W. H. (2012). IPEG Pharmaco-EEG Guidelines Committee: guidelines for the recording and evaluation of pharmaco-EEG data in man: the International Pharmaco-EEG Society (IPEG). Neuropsychobiology 66, 201-220. doi: 10.1159/000343478

John, E. R., Ahn, H., Prichep, L. S., Trepetin, M., Brown, D., Kaye, H., et al. (1980). Developmental equations for the electroencephalogram. Science 210, 1255-1258. doi: 10.1126/science.7434026

Killick, R., Ribe, E. M., Al-Shawi, R., Malik, B., Hooper, C., Fernandes, C., et al. (2012). Clusterin regulates $\beta$-amyloid toxicity via Dickkopf-1-driven induction of the wnt-PCP-JNK pathway. Mol. Psychiatry. doi: 10.1038/mp.2012.163 [Epub ahead of print].

Klimesch, W. (1999). EEG alpha and theta oscillations reflect cognitive and memory performance: a review and analysis. Brain Res. Brain Res. Rev. 29, 169-195. doi: 10.1016/S0165-0173(98)00056-3

Klimesch, W. (2012). Alpha-band oscillations, attention, and controlled access to stored information. Trends Cogn. Sci. 16, 606-617. doi: 10.1016/j.tics.2012.10.007

Knyazev, G. G. (2012). Extraversion and anterior vs. posterior DMN activity during self-referential thoughts. Front. Hum. Neurosci. 6:348. doi: 10.3389/fnhum.2012.00348

Koenig, T., Prichep, L., Dierks, T., Hubl, D., Wahlund, L. O., John, E. R., et al. (2005). Decreased EEG synchronization in Alzheimer's disease and mild cognitive impairment. Neurobiol. Aging 26, 165-171. doi: 10.1016/j.neurobiolaging.2004.03.008

Lambert, J. C., Heath, S., Even, G., Campion, D., Sleegers, K., Hiltunen, M., et al. (2009). Genome-wide association study identifies variants at CLU and CR1 associated with Alzheimer's disease. Nat. Genet. 41, 1094-1099. doi: 10.1038/ng.439 
Lancaster, T. M., Baird, A., Wolf, C., Jackson, M. C., Johnston, S. J., Donev, R., et al. (2011). Neural hyperactivation in carriers of the Alzheimer's risk variant on the clusterin gene. Eur. Neuropsychopharmacol. 21, 880-884. doi: 10.1016/j.euroneuro.2011.02.001

Lee, T. W., Yu, Y. W., Hong, C. J., Tsai, S. J., Wu, H. C., and Chen, T. J. (2012). The influence of apolipoprotein E Epsilon4 polymorphism on qEEG profiles in healthy young females: a resting EEG study. Brain Topogr. 25, 431-442. doi: 10.1007/s10548-012-0229-y

Lehtovirta, M., Partanen, J., Könönen, M., Hiltunen, J., Helisalmi, S., Hartikainen, P., et al. (2000). A longitudinal quantitative EEG study of Alzheimer's disease: relation to apolipoprotein E polymorphism. Dement. Geriatr. Cogn. Disord. 11, 29-35. doi: 10.1159/000017210

Levy-Lahad, E., Wasco, W., Poorkaj, P., Romano, D., Oshima, J., Pettingell, W., et al. (1995). Candidate gene for the chromosome 1 familial Alzheimer's disease locus. Science 269, 973-977. doi: 10.1126/science.7638622

Ling, I.-F., Bhongsatiern, J., Simpson, J. F., Fardo, D. W., and Estus, S. (2012). Genetics of clusterin isoform expression and Alzheimer's disease risk. PLoS ONE 7:e33923. doi: 10.1371/journal.pone.0033923

Lizio, R., Vecchio, F., Frisoni, G. B., Ferri, R., Rodriguez, G., and Babiloni, C. (2011). Electroencephalographic rhythms in Alzheimer's disease. Int. J. Alzheimers Dis. 2011, 927573. doi: 10.4061/2011/927573

Masdeu, J. C., Kreisl, W. C., and Berman, K. F. (2012). The neurobiology of Alzheimer disease defined by neuroimaging. Curr. Opin. Neurol. 25, 410-420. doi: 10.1097/WCO.0b013e3283557b36

Moretti, D. V., Babiloni, F., Carducci, F., Cincotti, F., Remondini, E., Rossini, P. M., et al. (2003). Computerized processing of EEG-EOG-EMG artifacts for multi-centric studies in EEG oscillations and event-related potentials. Int. J. Psychophysiol. 47, 199-216. doi: 10.1016/S0167-8760(02)00153-8

Moretti, D. V., Frisoni, G. B., Binetti, G., and Zanetti, O. (2011). Anatomical substrate and scalp EEG markers are correlated in subjects with cognitive impairment and Alzheimer's disease. Front. Psychiatry 1:152. doi: 10.3389/fpsyt.2010.00152

Moretti, D. V., Miniussi, C., Frisoni, G. B., Geroldi, C., Zanetti, O., Binetti, G., etal. (2007). Hippocampal atrophy and EEG markers in subjects with mild cognitive impairment. Clin. Neurophysiol. 118, 2716-2729. doi: 10.1016/j.clinph.2007.09.059

Moretti, D. V., Prestia, A., Fracassi, C., Binetti, G., Zanetti, O., and Frisoni, G. B. (2012a). Specific EEG changes associated with atrophy of hippocampus in subjects with mild cognitive impairment and Alzheimer's disease. Int. J. Alzheimers Dis. 2012, 253153. doi: 10.1155/2012/253153

Moretti, D. V., Zanetti, O., Binetti, G., and Frisoni, G. B. (2012b). Quantitative EEG markers in mild cognitive impairment: degenerative versus vascular brain impairment. Int. J. Alzheimers Dis. 2012, 917537. doi: 10.1155/2012/917537

Palop, J. J., and Mucke, L. (2009). Epilepsy and cognitive impairments in Alzheimer disease. Arch. Neurol. 66, 435-440. doi: 10.1001/archneurol.2009.15

Pfurtscheller, G., and Lopes da Silva, F. H. (1999). Event-related EEG/MEG synchronization and desynchronization: basic principles. Clin. Neurophysiol. 110, 1842-1857. doi: 10.1016/S1388-2457(99)00141-8

Pfurtscheller, G., Neuper, C., and Krausz, G. (2000). Functional dissociation of lower and upper frequency mu rhythms in relation to voluntary limb movement. Clin. Neurophysiol. 111, 1873-1879. doi: 10.1016/S1388-2457(00)00428-4

Ponomareva, N. V., Goltsov, A. Y., Kunijeva, S. S., Scheglova, N. S., Malina, D. D., Mitrofanov, A. A., et al. (2012). Age- and genotype-related neurophysiologic reactivity to oxidative stress in healthy adults. Neurobiol. Aging 33, 839.e11839.e21. doi: 10.1016/j.neurobiolaging.2011.11.013

Ponomareva, N. V., Korovaitseva, G. I., and Rogaev, E. I. (2008). EEG alterations in non-demented individuals related to apolipoprotein E genotype and to risk of Alzheimer disease. Neurobiol. Aging 29, 819-827. doi: 10.1016/j.neurobiolaging.2006.12.019

Prichep, L. S., John, E. R., Ferris, S. H., Rausch, L., Fang, Z., Cancro, R., et al. (2006). Prediction of longitudinal cognitive decline in normal elderly with subjective complaints using electrophysiological imaging. Neurobiol. Aging 27, 471-481. doi: 10.1016/j.neurobiolaging.2005.07.021

Rogaev, E. I. (1999). Genetic factors and a polygenic model of Alzheimer's disease. Genetika 35, 1558-1571.

Rogaev, E. I., Sherrington, R., Rogaeva, E. A., Levesque, G., Ikeda, M., Liang, Y., et al. (1995). Familial Alzheimer's disease in kindreds with missense mutations in a gene on chromosome 1 related to the Alzheimer's disease type 3 gene. Nature 376, 775-778. doi: 10.1038/376775a0
Saunders, A. M., Strittmatter, W. J., Schmechel, D., George-Hyslop, P. H., PericakVance, M. A., Joo, S. H., et al. (1993). Association of apolipoprotein E allele $\varepsilon 4$ with late-onset familial and sporadic Alzheimer's disease. Neurology 43, 1467-1472. doi: 10.1212/WNL.43.8.1467

Schliebs, R., and Arendt, T. (2011). The cholinergic system in aging and neuronal degeneration. Behav. Brain Res. 221, 555-563. doi: 10.1016/j.bbr.2010. 11.058

Schmechel, D. E., Saunders, A. M., Strittmatter, W. J., Crain, B. J., Hulette, C. M., Joo, C. H., et al. (1993). Increased amyloid $\beta$-peptide deposition in cerebral cortex as a consequence of an apolipoprotein E genotype in late-onset Alzheimer disease. Proc. Natl. Acad. Sci. U.S.A. 90, 9649-9653. doi: 10.1073/pnas.90.20.9649

Sherrington, R., Rogaev, E. I., Liang, Y., Rogaeva, E. A., Levesque, G., Ikeda, M., et al. (1995). Cloning of a gene bearing missense mutations in early-onset familial Alzheimer's disease. Nature 375, 754-760. doi: 10.1038/375754a0

Song, F., Poljak, A., Crawford, J., Kochan, N. A., Wen, W., Cameron, B., et al. (2012). Plasma apolipoprotein levels are associated with cognitive status and decline in a community cohort of older individuals. PloS ONE 7:e34078. doi: 10.1371/journal.pone.0034078

Spielberger, C. D. (1983). Manual for the State-Trait Anxiety Inventory: STAI (Form Y). Palo Alto: Consulting Psychologists Press.

Stam, C. J., van der Made, Y., Pijnenburg, Y. A., and Scheltens, P. (2003). EEG synchronization in mild cognitive impairment and Alzheimer's disease. Acta Neurol. Scand. 108, 90-96. doi: 10.1034/j.1600-0404.2003.02067.x

Steriade, M. (2006). Grouping of brain rhythms in corticothalamic systems. Neuroscience 137, 1087-1106. doi: 10.1016/j.neuroscience.2005.10.029

Steriade, M., and Llinás, R. R. (1988). The functional states of the thalamus and the associated neuronal interplay. Physiol. Rev. 68, 649-742.

Suslina, Z. A. (2012). Neurology at the border of centuries: achievements and prospects. Vestn. Ross. Akad. Med. Nauk 57-65.

Tang, M., Li, J., Liu, B., Song, N., Wang, Z., and Yin, C. (2013). Clusterin expression and human testicular seminoma. Med. Hypotheses 81, 635-637. doi: 10.1016/j.mehy.2013.07.019

Tatum, W. O., Dworetzky, B. A., and Schomer, D. L. (2011). Artifact and recording concepts in EEG. J. Clin. Neurophysiol. 28, 252-263. doi: 10.1097/WNP.0b013e31821c3c93

Thambisetty, M., Beason-Held, L. L., An, Y., Kraut, M., Nalls, M., Hernandez, D. G., et al. (2013). Alzheimer risk variant CLU and brain function during aging. Biol. Psychiatry 73, 399-405. doi: 10.1016/j.biopsych.2012.05.026

Tsuno, N., Shigeta, M., Hyoki, K., Kinoshita, T., Ushijima, S., Faber, P. L., et al. (2002). Spatial organization of EEG activity from alertness to sleep stage 2 in old and younger subjects. J. Sleep Res. 11, 43-51. doi: 10.1046/j.1365-2869.2002. 00288.x

van Beijsterveldt, C. E., Molenaar, P. C., de Geus, E. J., and Boomsma, D. I. (1996). Heritability of human brain functioning as assessed by electroencephalography. Am. J. Hum. Genet. 58, 562-573.

Van der Hiele, K., Bollen, E. L., Vein, A. A., Reijntjes, R. H., Westendorp, R. G., van Buchem, M. A., et al. (2008). EEG markers of future cognitive performance in the elderly. J. Clin. Neurophysiol. 25, 83-89. doi: 10.1097/WNP.0b013e31816a5b25

Versavel, M., Leonard, J. P., and Herrmann, W. M. (1995). Standard operating procedure for registration and computer-supported evaluation of pharmaco-EEG data. Neuropsychobiology 32, 166-170. doi: 10.1159/000119230

Vlassenko, A. G., Vaishnavi, S. N., Couture, L., Sacco, D., Shannon, B. J., Mach, R. H., et al. (2010). Spatial correlation between brain aerobic glycolysis and amyloid- $\beta$ (A $\beta$ ) deposition. Proc. Natl. Acad. Sci. U.S.A. 107, 17763-17767. doi: 10.1073/pnas.1010461107

Winterer, G., Mahlberg, R., Smolka, M. N., Samochowiec, J., Ziller, M., Rommelspacher, H. P., et al. (2003). Association analysis of exonic variants of the $\operatorname{GABA}(\mathrm{B})$-receptor gene and alpha electroencephalogram voltage in normal subjects and alcohol-dependent patients. Behav. Genet. 33, 7-15. doi: 10.1023/A:1021043315012

Conflict of Interest Statement: The authors declare that the research was conducted in the absence of any commercial or financial relationships that could be construed as a potential conflict of interest.

Received: 10 September 2013; paper pending published: 29 September 2013; accepted: 19 November 2013; published online: 13 December 2013.

Citation: Ponomareva N, Andreeva T, Protasova M, Shagam L, Malina D, Goltsov A, Fokin V, Mitrofanov A and Rogaev E (2013) Age-dependent effect of Alzheimer's risk 
variant of CLU on EEG alpha rhythm in non-demented adults. Front. Aging Neurosci. 5:86. doi: 10.3389/fnagi.2013.00086

This article was submitted to the journal Frontiers in Aging Neuroscience.

Copyright (C) 2013 Ponomareva, Andreeva, Protasova, Shagam, Malina, Goltsov, Fokin, Mitrofanov and Rogaev. This is an open-access article distributed under the terms of the
Creative Commons Attribution License (CC BY). The use, distribution or reproduction in other forums is permitted, provided the original author(s) or licensor are credited and that the original publication in this journal is cited, in accordance with accepted academic practice. No use, distribution or reproduction is permitted which does not comply with these terms. 